\title{
Corrigendum
}

\section{Theropod dinosaur trackways from the Lower Cretaceous of the Chacarilla Formation, Chile. Revista Geológica de Chile 35 (1): 175-184.}

\author{
Karen Moreno ${ }^{1,2}$ \\ 1 Université Paul Sabatier, Laboratoire d'Anthropologie Moléculaire et Imagerie de Synthèse (CNRS-FRE 2960), 39 Allée Jules \\ Guesde, 31073, Toulouse, France. \\ kmoreno@cict.fr \\ 2 Universidad Austral de Chile, Instituto de Geociencias, Laboratorio de Paleoecología, Valdivia, Chile.
}

English: I apologize for the involuntary errors shown at Table 1 (Rubilar-Rogers et al., 2008). Values for the calculation of the speed in $\mathrm{cm} / \mathrm{s}$, $\mathrm{km} / \mathrm{h}$ and $\mathrm{S} / \mathrm{H}$ now take account of the correction factor 4.9 for calculating the hip height of large theropods from footprint length and not 4.5 as it was published in the original article. Also, the formula for calculating the speed given in the legend was corrected, since it showed the parameter L (footprint length) instead of S (stride length). The modification of the values does not change the sense of either the discussion nor the conclusions on the original article, but they remain important for the understanding of the dinosaur speed formula.

These mistakes were found by the schoolteacher of Physics, Martín Ghizzoni, to whom I'm deeply grateful.
Castellano: Me disculpo por el error involuntario cometido en la Tabla 1 (Rubilar-Rogers et al., 2008). Valores para el cálculo de la velocidad en $\mathrm{cm} / \mathrm{s}, \mathrm{km} / \mathrm{h}$ y $\mathrm{S} / \mathrm{H}$ ahora toman en cuenta el factor 4,9 para el cálculo de la altura de cadera a partir del largo de la huella, y no 4,5 como se había publicado en el artículo original. Además, la fórmula para calcular la velocidad dada en la leyenda fue corregida, debido a que presentaba el parámetro L (largo de la huella) en vez de S (largo de la zancada, distancia entre dos huellas del mismo pie). La modificación de los valores no cambia el sentido de la discusión ni las conclusiones del artículo original, pero es importante para la comprensión del uso de la fórmula para calcular la velocidad en dinosaurios.

Estos errores fueron encontrados por el profesor de Física, Martín Ghizzoni, a quien expreso mi más sincero agradecimiento. 
TABLE 1. MEASUREMENTS OF THE FOOTPRINTS AT SITE III OF THE CHACARILLA FORMATION.

\begin{tabular}{|c|c|c|c|c|c|c|c|c|c|c|c|c|}
\hline Trackway & $\mathbf{N}$ & $\begin{array}{c}\mathrm{L} \\
(\mathrm{cm})\end{array}$ & $\begin{array}{c}W \\
(\mathrm{~cm})\end{array}$ & II/L & IV/L & $\begin{array}{c}P \\
(\mathrm{~cm})\end{array}$ & $\begin{array}{l}\mathbf{A} \\
\left({ }^{\circ}\right)\end{array}$ & $\begin{array}{c}\mathrm{S} \\
(\mathrm{cm})\end{array}$ & $\begin{array}{c}\mathbf{H} \\
(\mathrm{cm})\end{array}$ & $\begin{array}{l}\text { Speed } \\
\mathrm{cm} / \mathrm{s}\end{array}$ & $\begin{array}{l}\text { Speed } \\
\mathrm{km} / \mathrm{h}\end{array}$ & $\mathbf{S} / \mathbf{H}$ \\
\hline \multicolumn{13}{|c|}{ Small theropod footprints } \\
\hline 17 & 6 & 23 & 21 & 0.2 & 0.2 & 116 & 180 & 232 & 104 & 3.1 & 11.2 & 2.2 \\
\hline $18 \dagger$ & 2 & 16 & 13 & 0.5 & 0.5 & 61 & - & - & 72 & - & - & - \\
\hline Average & & 19.5 & 17 & 0.3 & 0.3 & 88.5 & 180 & 232 & 88 & 3.1 & 11.2 & 2.2 \\
\hline St. Dev. & & 5 & 6 & 0.2 & 0.2 & 39 & - & - & - & - & - & - \\
\hline \multicolumn{13}{|c|}{ Large theropod footprints } \\
\hline $3 \dagger$ & 8 & 33 & 21 & 0.5 & 0.5 & 119 & 180 & 234 & 162 & 1.8 & 6.6 & 1.4 \\
\hline $4 \dagger$ & 8 & 34 & 22 & 0.5 & 0.5 & 120 & 180 & 241 & 167 & 1.9 & 6.7 & 1.4 \\
\hline 5 & 6 & 31 & 29 & 0.3 & 0.3 & 117 & 178 & 234 & 152 & 2.0 & 7.1 & 1.5 \\
\hline 6 & 1 & 55 & 37 & 0.3 & 0.3 & - & 176 & - & 270 & - & - & - \\
\hline 8 & 3 & 50 & 44 & 0.2 & 0.3 & 155 & 176 & 285 & 245 & 1.6 & 5.7 & 1.2 \\
\hline 10 & 6 & 35 & 25 & 0.3 & 0.3 & 115 & 180 & 230 & 172 & 1.7 & 6.0 & 1.3 \\
\hline 12 & 9 & 40 & 36 & 0.3 & 0.3 & 132 & 180 & 252 & 196 & 1.7 & 6.0 & 1.3 \\
\hline 13 & 5 & 63 & 46 & 0.3 & 0.3 & 138 & 180 & 265 & 309 & 1.1 & 3.8 & 0.9 \\
\hline 15 & 7 & 60 & 45 & 0.3 & 0.3 & 160 & 180 & 307 & 294 & 1.4 & 5.2 & 1.0 \\
\hline 16 & 6 & 65 & 45 & 0.3 & 0.3 & 125 & 180 & 273 & 319 & 1.1 & 3.9 & 0.9 \\
\hline 22 & 8 & 60 & 53 & 0.3 & 0.3 & 152 & 180 & 322 & 294 & 1.6 & 5.6 & 1.1 \\
\hline 24 & 2 & 28 & 15 & 0.3 & 0.3 & 90 & - & - & 137 & - & - & - \\
\hline 25 & 2 & 50 & 45 & 0.3 & 0.3 & 145 & - & - & 245 & - & - & - \\
\hline $26^{*}$ & 7 & 60 & 50 & 0.2 & 0.2 & 172 & 175 & 330 & 294 & 1.6 & 5.9 & 1.1 \\
\hline Average & & 47 & 37 & 0.3 & 0.3 & 134 & 179 & 270 & 232 & 1.6 & 5.7 & 1.2 \\
\hline St. Dev. & & 13 & 12 & 0.1 & 0.1 & 23 & 2 & 36 & 66 & 0.3 & 1.1 & 0.2 \\
\hline
\end{tabular}

N: number of footprints; L: footprint length; W: footprint width; II/L: digit II-footprint length ratio; IV/L: digit IV- footprint length ratio; P: pace length; A: pace angle; S: stride length; H: hip height calculated using the correction factor of Thulborn (1984): either 4.5 or 4.9 times the footprint length for small or large theropods respectively. Speed is calculated with the formula developed by Alexander (1976) in MKS units; Speed: 2.817 S1.67H-1.17; $\uparrow$ : Theropods with morphology A, in which the length of digits II and IV is half the length of digit III; *: Length of the footprint without considering the metatarsal impression.

\section{References}

Rubilar-Rogers, D.; Moreno, K.; Blanco, N.; Calvo, J.O. 2008. Theropod dinosaur trackways from the Lower Cretaceous of the Chacarilla Formation, Chile. Revista Geológica de Chile 35 (1): 175-184. 